Uniciencia. Vol. 29, No. 1, pp. 1-15. Enero, 2015.

ISSN Electrónico: 2215-3470

URL: www.revistas.una.ac.cr/uniciencia

Email: revistauniciencia@una.cr

DOI: http://dx.doi.org/10.15359/ru.29-1.1

\title{
Comparación del crecimiento de machos y hembras de la tilapia Oreochromis Niloticus cultivadas en jaulas
}

\author{
Comparison of the growth of males and females of tilapia Oreochromis Niloticus cultured \\ in cages
}

Carlos Alvarado-Ruiz
calvaradoruiz@ina.ac.cr
Instituto Nacional de Aprendizaje INA
Núcleo Náutico Pesquero
Costa Rica

Fecha de recepción del artículo: 10 de setiembre de 2012.

Fecha de revisión del artículo: 3 de febrero de 2014.

Fecha de aprobación del artículo: 9 de abril de 2014.

\section{Resumen}

Con el fin de evaluar el desempeño productivo y el potencial de engorde de la tilapia $O$. niloticus sin reversar, se evaluó el crecimiento de machos y hembras cultivadas en forma separada en jaulas flotantes. El experimento se realizó por un periodo de 150 días, para ello se utilizó una población de 4836 alevines de $26.2 \pm 19.7 \mathrm{~g}$, peces que fueron engordados hasta peso de diferenciación sexual a $119.3 \pm 56.5 \mathrm{~g}$, para luego ser separados por sexo y engordados en jaulas flotantes hasta un peso final promedio de $387.0 \pm 128.9 \mathrm{~g}$ (cosecha) y determinar así diferencias en crecimiento.

La distribución de pesos de la población sexo indefinida mostró una disminución en su coeficiente de variación (CV) de $75.13 \%$ a $31.49 \%$ y $30.22 \%$ para los machos y hembras respectivamente, lo cual es un indicador de que la distribución de tallas de la poblaciones de peces tendió a normalizarse a lo largo del ciclo de cultivo. Diferencias significativas $(\mathrm{P}<=0.05)$ para el peso de cosecha $429.2 \pm 135.1 \mathrm{~g}$ y $339.65 \pm 102.64 \mathrm{~g}$, y para la tasa de crecimiento $2.74 \pm 0.86 \mathrm{~g} \mathrm{día}^{-1}$ y $2.18 \pm$ $0.68 \mathrm{~g} \mathrm{día}^{-1}$ fueron determinadas entre machos y hembras respectivamente.

El error estándar de la estimación de la diferencia de promedios de peso para machos y hembras correspondió a $3.68 \mathrm{~g}$ y la estimación de la diferencia de pesos de los machos con respecto a las hembras correspondió a $89.62 \pm 7.23$ g para un intervalo de confianza del 95\%. Los machos mostraron un mejor desempeño productivo para peso de cosecha y tasa de crecimiento.

Palabras clave: Sexo indiferenciado, reversión de sexo, larvas, jaulas.

\begin{abstract}
With the purpose of evaluating productive performance and potential weight gain capacity of snapper $O$. Niloticus without reversing, we evaluated the growth of males and females raised in floating cages in captivity grouped by gender. The experiment was conducted for a term of 150 days. We used a simple 4836 alevins of $26.2 \pm 19.7 \mathrm{~g}$, fish that were nourished until they visibly reached sexual differentiation to $119.3 \pm 56.5 \mathrm{~g}$ to be separated by sex and nourished in floating cages to a final average weight of $387.0 \pm 128.9 \mathrm{~g}$ (harvest)to determine differences in growth.
\end{abstract}

Carlos Alvarado Ruiz

Artículo protegido por licencia Creative Commons: BY-NC-ND / Protected by Creative Commons: BY-NC-ND

Revista de acceso y publicación gratuita/ Access and publication in Uniciencia is totally no fee. 
The weight distribution of the undefined gender sample showed a decrease in the coefficient of variation (CV) of $75.13 \%$ to 31.49 and 30.22 for males and females respectively. This indicates that the distribution of sizes of the samples tended to become normal during the cycle of harvest. Significant differences $(\mathrm{P}=0.05)$ for the weight of harvest $429.2 \pm 135.1 \mathrm{~g}$ and $339.65 \pm 102.64 \mathrm{~g}$.

For the growth rate $2.74 \pm 0.86 \mathrm{~g}$ day y $2.18 \pm 0.68 \mathrm{~g}$ day were determined between males and females respectively. The standard error of the estimation of the difference of average weight between males and females was $89.62 \pm 7.23 \mathrm{~g}$ for an accuracy level of $95 \%$. Males displayed better growth rate and weight gain for harvest.

Key words: undifferentiated sex, sex reversal, larvae, cages.

La frecuencia relativa de machos y hembras se denomina proporción de sexos; durante las primeras etapas de la vida de los peces esta proporción, usualmente, es 1:1. Algunas condiciones particulares, como la edad de maduración sexual y la mortalidad de los individuos, pueden causar un desbalance en esta proporción (Gjedrem, 2005).

Para el caso de líneas puras de tilapia es de esperar que, en condiciones normales de reproducción, la progenie guarde una proporción de 1:1 por lo que el 50\% de la población de larvas en un desove corresponderá a hembras y el otro restante a machos. Los machos tienen un crecimiento mayor que las hembras, debido a que estos utilizan la energía extraída del alimento en su crecimiento somático, mientras que las hembras la utilizan en la producción de gametos para desove (Chakraborty \& Banerjee, 2010).

Poblaciones de solo machos en tilapias pueden ser obtenidas a través de hibridación, por ejemplo, al cruzar machos $\boldsymbol{O}$. aureus que cuentan con cromosomas $\mathrm{ZZ}$, con hembras O. niloticus cuyos cromosomas son $\mathrm{XX}$, se generarán progenies híbridas de machos $\mathrm{XZ}$ (Dunham et al. 2000). Otra alternativa es mediante la reversión de sexos, la obtención de poblaciones monosexo consiste en el suministro de alimento con andrógenos (17 alfa metil testosterona) a poblaciones de alevines sexualmente indiferenciados (Mair, 1995). El suministro de alimento hormonado causará que las hembras manifiesten un morfo tipo de macho, mejorando así su desempeño productivo.

La generación de tilapias denominadas supermachos (YY) es otra tecnología utilizada para producir machos de tilapias, estas se obtienen a partir del suministro de estrógenos (ß-estradiol) a juveniles de tilapia indiferenciados, lo que producirá neohembras

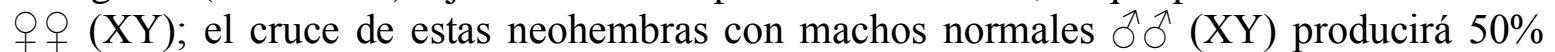

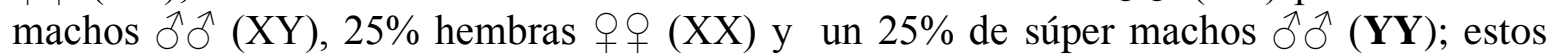
últimos, al ser cruzados con hembras normales $(\mathrm{XX})$, producirán solo machos en la progenie (Mair,1995).

El engorde de poblaciones de tilapia mono sexo (machos) es una práctica común que se utiliza en todo el mundo, debido a los problemas que generan las hembras en la población: primero, por aporte de alevines al estanque y segundo, por su menor desempeño productivo (menor crecimiento). Las hembras de tilapia alcanzan su madurez sexual a pesos entre los 30 g y 40 g (Bujhel, 2000), y la presencia de crías causa un problema 
productivo, ya que las hembras estarán produciendo progenie cada dos semanas, provocando un desbalance en el inventario de peces dentro del estanque, y los nuevos reclutas consumirán parte del alimento destinado al engorde de los peces de interés.

Algunas alternativas para controlar la aparición de nuevos peces sin el uso de hormonas han sido implementadas. Entre estas están: La selección manual separando hembras, la utilización de controladores biológicos (depredadores) para las crías y el cultivo de tilapias en jaulas para evitar la reproducción (Mair, 1995).

La producción de alevines sexo reversados es realizada por productores privados y por algunas instituciones estatales; no obstante, para pequeños productores de zonas alejadas a los centros de distribución, la compra y los costos asociados al transporte de los alevines se traduce en inversiones onerosas que desestimulan la producción de esta especie, por lo que la producción de alevines sin reversar para su engorde, representa una alternativa para solventar estos inconvenientes.

La utilización de poblaciones de sexo mixto no es una estrategia de uso frecuente en el engorde de tilapias, al menos en Costa Rica, por lo que no se cuenta con datos referentes a la tasa de crecimiento por sexo, ni el comportamiento de la distribución de tamaños de los machos y hembras cultivadas en estas condiciones.

El presente estudio tiene como objetivo evaluar el desempeño productivo de machos y hembras de la especies $\boldsymbol{O}$. niloticus cultivadas en jaulas flotantes y por sexo separado, y determinar la existencia o no de diferencias estadísticas significativas para el peso final de engorde y para las tasas de crecimiento por sexo. Se pretende contar con datos de producción para machos y hembras en cultivo separado, con el fin de valorar la factibilidad de su engorde en jaulas.

\section{Material y métodos}

De enero a julio del 2008 y por un periodo de 150 días se realizó el engorde de una población de alevines sin reversar de la especie de tilapia $\boldsymbol{O}$. niloticus. Los alevines fueron colectados de varias hapas de reproducción de una unidad de producción de alevines ubicada en Tilarán-Guanacaste, Costa Rica, denominada Centro Acuícola Viejo Arenal, con localización en coordenadas latitud $=10^{\prime \prime} 30^{\prime} 12^{\prime \prime} \mathrm{N}$ y longitud $=84^{\prime \prime} 55^{\prime} 30^{\prime \prime} \mathrm{O}$, elevación $600 \mathrm{~m}$. El engorde de los peces fue realizado en el mismo centro acuícola. Durante el cultivo se realizó el sexado de los peces y su engorde en forma separada, utilizando para ello jaulas flotantes de $15 \mathrm{~m}^{3}$.

\section{Unidad experimental}

Se usó un estanque de tierra con un área de $1500 \mathrm{~m}^{2}$ con una profundidad de $0.5 \mathrm{~m}$ en la parte más baja y $1.5 \mathrm{~m}$ en la zona más profunda. El estanque contó con una toma de agua a través de tubería plástica de PVC de 8.0 pulgadas de diámetro; el drenaje fue construido mediante un codo y tubo de iguales características; el nivel de profundidad promedio del estaque fue de $0.8 \mathrm{~m}$. 


\section{Características de alevines}

Una población de 4177 alevines de 105 días de edad y sin reversar con 26.2 g de peso promedio fueron engordados en tres jaulas flotantes de $15 \mathrm{~m}^{3}$ de $1^{1 / 2}$ pulgada de luz de malla, hasta su diferenciación sexual a los $119.3 \pm 56.5 \mathrm{~g}$, para una densidad inicial de 92.8 peces $\mathrm{m}^{3}$ y una densidad final de $13.0 \mathrm{~kg} \mathrm{~m}^{3}$. Para peces de $18.0 \mathrm{~g}$ se recomiendan siembras de 500 peces $\mathrm{m}^{3}$ para una densidad máxima de $9.0 \mathrm{~kg} \mathrm{~m}^{3}$ (Schmittou, 1991); mientras que para rangos de peso de $16.0 \mathrm{~g}$ a $105.0 \mathrm{~g}$ se sugieren densidades de siembra máximas de $34.3 \mathrm{~kg} \mathrm{~m}^{3}$, donde la densidad final depende del nivel de recambio de agua con que cuente el lago o reservorio de agua (Nunes, 2010).

\section{Sexado de población}

Las hembras de la tilapia alcanzan su madurez sexual entre los $20.0 \mathrm{~g}$ y $40.0 \mathrm{~g}$; sin embargo, sus huevos pueden ser reabsorbidos antes de que estas desoven por primera vez (Bhujel, 2000). Otros autores consideran que la madurez sexual se presenta a pesos mayores entre los $80.0 \mathrm{~g}$ y $100.0 \mathrm{~g}$, lo que corresponde a una edad entre 5.0 y 6.0 meses (Alicorp, 2004). En este experimento se realizó la separación por sexo a los $119.37 \pm 56.5 \mathrm{~g}$ promedio poblacional, para ello se utilizó como referencia la morfología de la papila urogenital descrita por Saavedra (2006). El sexado se realizó mediante observación y palpaje de la papila urogenital; para el caso de los peces más pequeños, se utilizo una lupa para magnificar el tamaño de la papila y así diferenciar certeramente su sexo (figura 1).
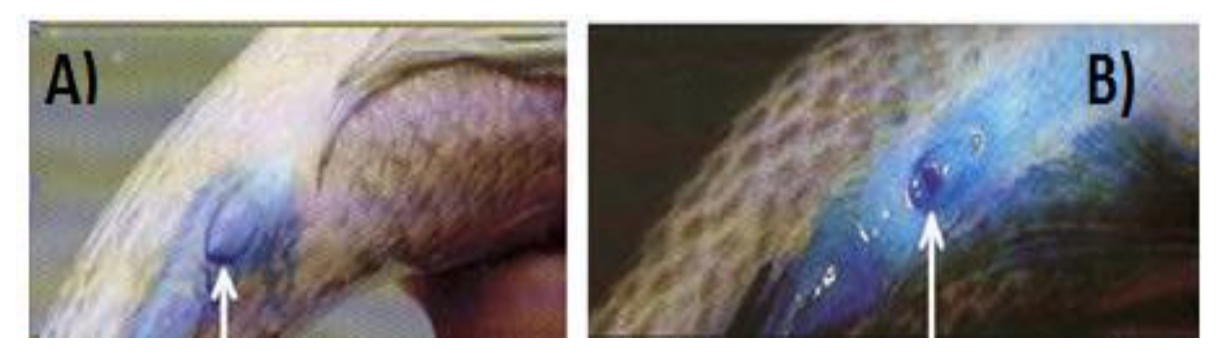

Figura 1.

Diferencias anatómicas de la papila urogenital de machos $\boldsymbol{A}$ y hembras $\boldsymbol{B}$.

Tomado de Saavedra, 2006, p. 4.

\section{Engorde por sexo separado}

Una vez separados como machos y hembras, se continuó el engorde hasta un peso promedio final de $387.0 \mathrm{~g} \pm 128.9$ (peso de cosecha). Para ello se utilizaron 9 jaulas flotantes (5 para los machos y 4 para las hembras) de $15 \mathrm{~m}^{3}$ de $1^{3 / 4}$ pulgadas de luz de malla, para una densidad máxima por jaulas de $13.0 \mathrm{~kg} \mathrm{~m}^{-3}$. Las densidades de siembra para tilapias en jaulas han sido determinadas por número de peces y kilos por metro cúbico, se establecen 
como topes de siembra máxima 80 peces $\mathrm{m}^{-3}$ para una densidad máxima de $150 \mathrm{~kg} \mathrm{~m}^{-3}$ (Schmittou, 1991), para pesos de $105 \mathrm{~g}$ a $500 \mathrm{~g}$ la densidad máxima a utilizar fluctúa entre $60 \mathrm{~kg} \mathrm{~m}^{-3}$ y $80.0 \mathrm{~kg} \mathrm{~m}^{-3}$ (Nunes, 2010). Para el caso de esta investigación las densidades utilizadas fueron mucho más bajas.

\section{Alimentación}

Se utilizó como referencia la tabla de alimentación para tilapias propuesta por Alicorp (2004), la cual suministra una tasa inicial del $4.5 \%$ y una final del 1.7\% de su peso corporal; se proyectó una tasa de crecimiento de $2.61 \mathrm{~g}_{\text {día }}{ }^{-1}$ con un factor de conversión alimenticia de 1.38; durante el periodo de engorde (108 días), se ajustó la cantidad de alimento al descontar los individuos muertos del inventario. El alimento se suministró de las 6:00 horas a las 15:00 horas, hasta completar la ración de alimento diaria.

\section{Mediciones de peso}

Previo a la siembra y durante el engorde se midió el peso individual de cada pez, para ello se utilizó una romana electrónica marca Ohaus 2000, con un grado de precisión de $( \pm 0.5 \mathrm{~g})$. Se realizaron tres mediciones de peso: la primera, al momento de la siembra; la segunda, durante el sexado y siembra en jaulas como machos y hembras y, la tercera al final del engorde. Los datos de peso y sexo fueron registrados y asociados a la fecha del censo poblacional.

\section{Capacidad de carga}

La densidad máxima de peces utilizada en las jaulas flotantes fue de $13.0 \mathrm{~kg} \mathrm{~m}^{-3} \mathrm{el}$ tamaño de las jaulas flotantes fue de $6.0 \mathrm{~m} \mathrm{~L} \mathrm{x} 3.0 \mathrm{~m} \mathrm{~A} \mathrm{x} 0.9 \mathrm{H} \mathrm{m}$ de profundidad, para un volumen efectivo de $15 \mathrm{~m}^{3}$. Se realizó el cultivo a un nivel de capacidad de carga relativamente bajo con respecto a lo recomendado por Schmittou (1991) y Nunes (2010). Esto, con el fin de no causar estrés ni perdidas de inventario por enfermedades o déficit de oxígeno en el sistema de cultivo.

\section{Crecimiento y relación de pesos}

Con los datos de peso individual de machos y hembras durante el periodo de engorde, se calculó la tasa absoluta de crecimiento para todo el periodo de engorde mediante la siguiente fórmula (Hopkins, 1992):

Tasa absoluta de crecimiento $=[($ Peso final $)-($ Peso inicial $)] /$ Días ciclo $\left(\mathrm{g} \mathrm{d}^{-1}\right)$

Mientras que la relación de peso de macho con respecto a la hembra, se calculó por medio de la siguiente fórmula: 
Uniciencia. Vol. 29, No. 1, pp. 1-15. Enero, 2015.

M-H/M = $[($ Peso promedio M - Peso promedio H) / (Peso promedio M)] X 100
$(\%)$
M: Macho
H: Hembra
Esta fórmula expresa cuánto más livianas (\%) son las hembras con respecto a los
machos y corresponde a una elaboración propia.

\section{Análisis de datos}

Con los datos de pesos individuales durante la siembra, sexado y cosecha, se realizaron los cálculos estadísticos básicos (promedio, desviación estándar, valores máximos, mínimos y curtosis) y se elaboraron las curvas de distribución poblacional. Se aplicó un análisis de variancia prueba de Tukey (ANDEVA) de una vía con un nivel de significancia de $\mathrm{P}=0.05$ para los datos de peso de cosecha y la tasa de crecimiento entre machos y hembras; se utilizó para ello el programa estadístico GenStat Discovery, versión 3 (Buysee et al., 2008). Se realizó la estimación del error estándar sobre el peso promedio de cosecha de machos y hembras, así como la estimación de diferencia de peso entre machos y hembras (IC 95\%).

La población de peces se clasificó de la siguiente forma:

- A-Población sexo indefinido

- B-Población diferenciada por sexo (macho/hembra)

- C-Población sexo separado bajo engorde.

\section{Resultados}

\section{Oxígeno disuelto}

Durante el periodo de engorde, los niveles de oxígeno se mantuvieron por encima de los $3.0 \mathrm{mg} \mathrm{L}^{-1}$, lo cual representa el nivel crítico para la tilapia. El valor promedio registrado de oxígeno fue de $6.1 \pm 0.57 \mathrm{mg} \mathrm{L}^{-1}$, con un máximo de 7.3 y un mínimo de 4.7 $\mathrm{mg} \mathrm{L}^{-1}$ (figura 2). Para el cultivo de la tilapia, la concentración mínima requerida para mantener un crecimiento normal y garantizar una baja mortalidad debe ser superior a los $3.0 \mathrm{mg} \mathrm{L}^{-1}$ (Alicorp, 2004). 
Uniciencia. Vol. 29, No. 1, pp. 1-15. Enero, 2015.

ISSN Electrónico: 2215-3470 URL: www.revistas.una.ac.cr/uniciencia

Email: revistauniciencia@una.cr

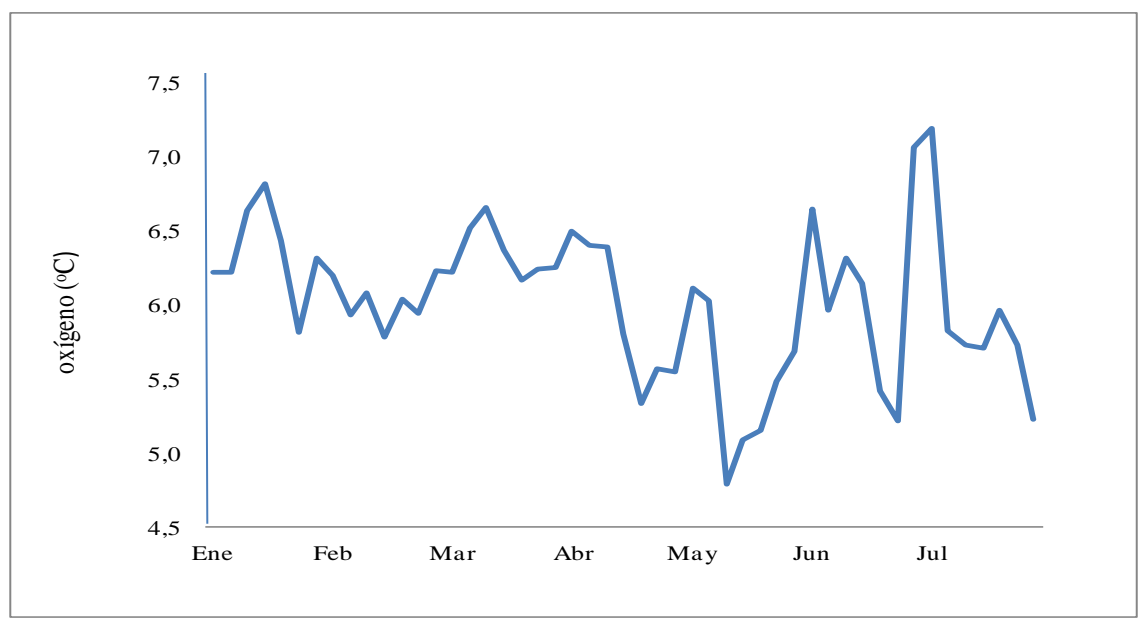

Figura 2.

Perfil de oxígeno ( $m g$ L-1) promedio en el estanque de engorde .

\section{Temperatura}

La temperatura promedio del estanque de producción fue de $28.0 \pm 1.0{ }^{\circ} \mathrm{C}$, con un máximo de $29.8{ }^{\circ} \mathrm{C}$ y un mínimo de $26.3{ }^{\circ} \mathrm{C}$. La temperatura mostró un incremento paulatino a lo largo del ciclo, con algunas disminuciones leves (figura 3).

El óptimo térmico para la reproducción y engorde de tilapia se ubica entre los 25.0 y $29.0{ }^{\circ} \mathrm{C}$ (Ridha y Cruz, 1998), por lo que el proceso de engorde de los machos y hembras tuvo lugar dentro de los rangos térmicos apropiados para esta especie.

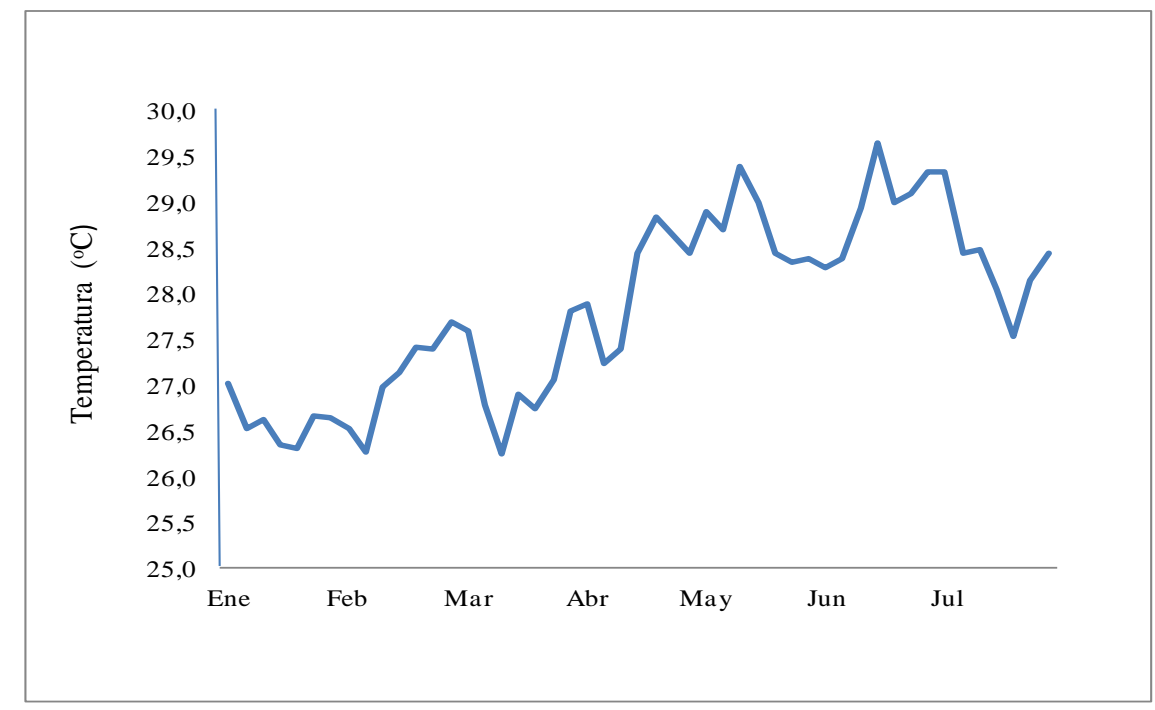

Figura 3.

Perfil de temperatura $\left({ }^{\circ} \mathrm{C}\right)$ del estanque utilizado para el engorde. 
Uniciencia. Vol. 29, No. 1, pp. 1-15. Enero, 2015.

ISSN Electrónico: 2215-3470

URL: www.revistas.una.ac.cr/uniciencia

Email: revistauniciencia@una.cr

DOI: http://dx.doi.org/10.15359/ru.29-1.1

\section{Población de alevines}

La población de alevines de sexo indefinido se caracterizó por presentar una distribución de pesos unimodal y asimétrica hacia la derecha (figura 4).

El peso promedio de los peces fue de 26.22 g con un máximo y un mínimo de 148.1 - 3.3 g respectivamente, con un alto coeficiente de variación (CV) de $75.13 \%$.

Se utilizó el dato de curtosis (Salvador \& Gallardo, 2003) para evaluar la forma de la campana de distribución de pesos de la población. El valor de curtosis de los alevines utilizados en esta investigación (3.94) fue un indicador de una distribución de pesos muy elevada (Leptocurtica) para los rangos de peso entre $13.3 \mathrm{~g}$ - $23.3 \mathrm{~g}$ (figura 4).

El alto valor de CV fue un indicador de que la población no se distribuyó normalmente; esto, debido a que los alevines fueron procedentes de desoves asincrónicos. Para la constitución de un lote de 4836 alevines se toman individuos procedentes de diferentes hapas de reproducción, con edades de nacimiento entre grupos de hasta 15 días de diferencia.

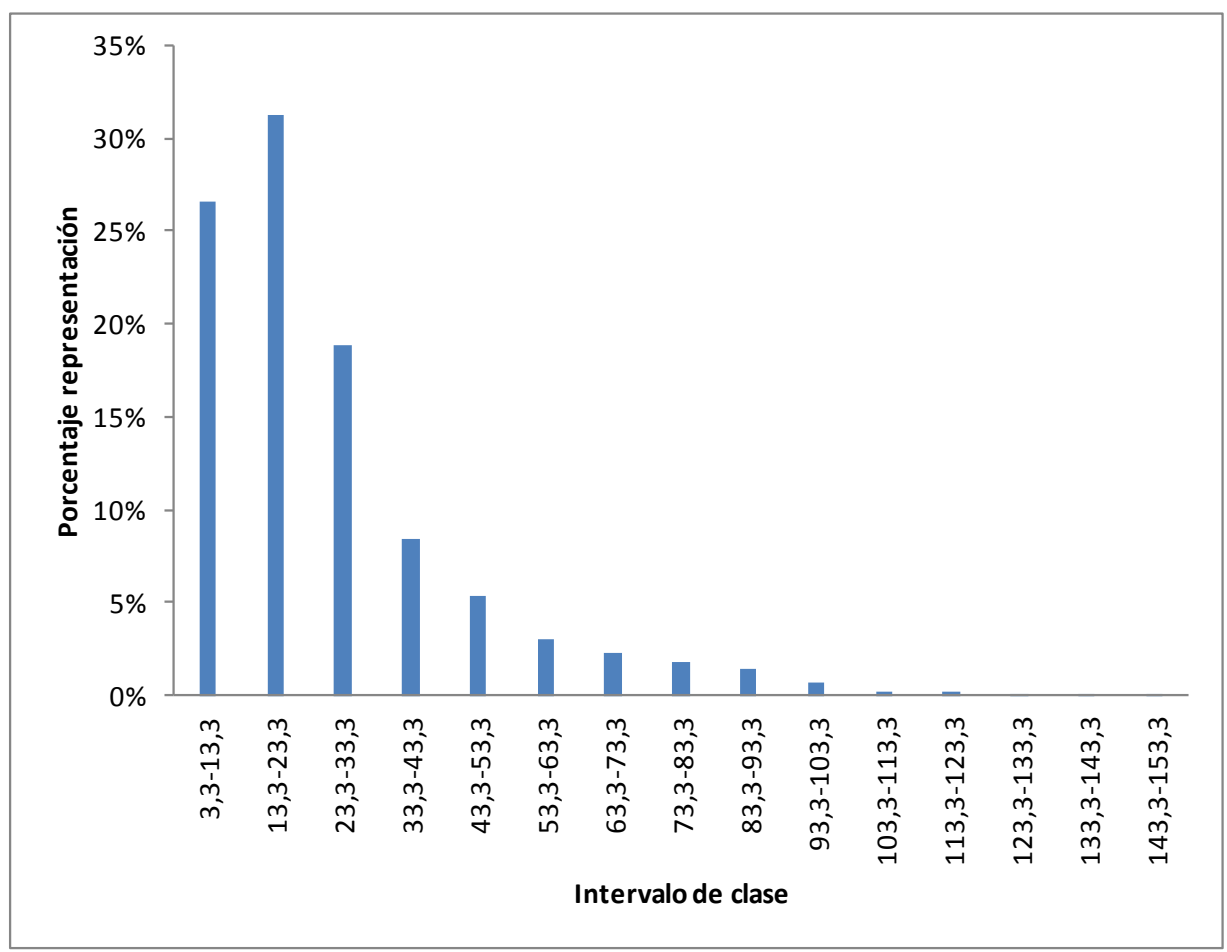

Figura 4.

Distribución de pesos de alevines sexo indefinido, durante la siembra en jaulas. 


\section{Sexado de peces}

La proporción de sexos obtenida después de la clasificación de la población fue de $52.8 \%$ y $47.2 \%$ de machos (1) y hembras (2) respectivamente. Los datos de peso derivaron dos curvas de distribución independientes, se presentó un traslape de pesos entre ambos sexos; el peso de los machos resultó superior al de las hembras; el $28.71 \%$ del total de pesos de las hembras se ubicó entre los $45.4 \mathrm{~g}$ y $105.4 \mathrm{~g}$ (figura 5).

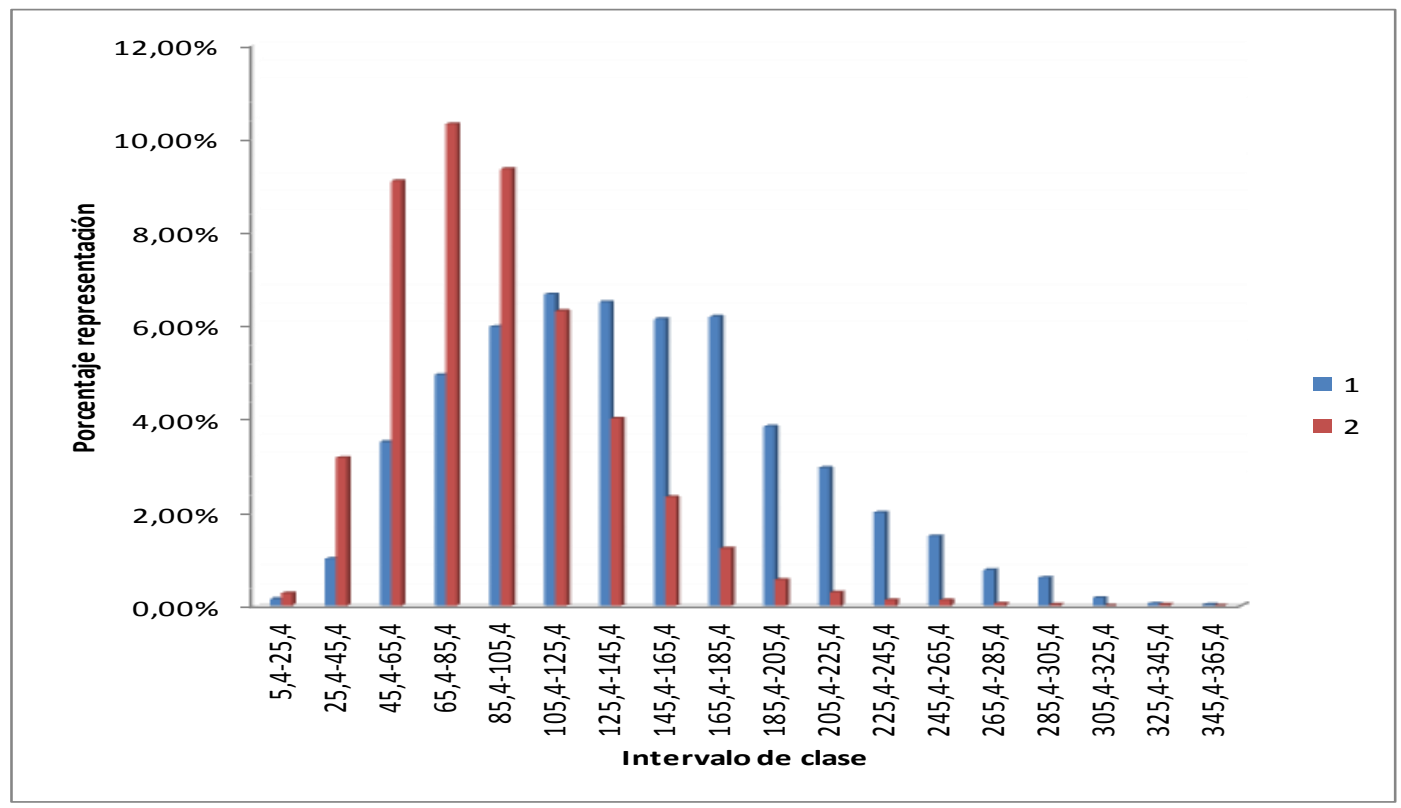

Figura 5.

Distribución de peso de machos (1) y hembras (2) al momento del sexado.

Para los machos se obtuvo un peso promedio de $142.8 \mathrm{~g}$ con un coeficiente de variación de $41.1 \%$, este último valor fue un indicador de que la población de pesos de los machos tendió a normalizarse con respecto a su población de origen. La curtosis de valor negativo -0.19 es un indicador de que la población de pesos de machos fue relativamente plana (Platicurtica) al momento de su sexado. Las hembras alcanzaron un peso promedio menor que los machos al momento del sexado con $93.05 \mathrm{~g}$, el coeficiente de variación de $42.5 \%$ fue mayor que el de los machos; sin embargo, también mejoró con respecto a su población de origen. El valor de curtosis en la población de hembras fue positiva con un valor de 1.99 (Leptocurtica) (tabla 1). 
Uniciencia. Vol. 29, No. 1, pp. 1-15. Enero, 2015.

URL: www.revistas.una.ac.cr/uniciencia

Email: revistauniciencia@una.cr
ISSN Electrónico: 2215-3470

DOI: http://dx.doi.org/10.15359/ru.29-1.1

Tabla 1

Resumen estadístico del peso de los machos y hembras, etapa sexado

\begin{tabular}{lccrrrr} 
Tipo de población & $\begin{array}{c}\text { Peso promedio en } \\
\text { gramos } \pm \mathrm{DE}\end{array}$ & $\mathrm{CV}$ & Moda & $\begin{array}{c}\text { Talla } \\
\text { máxima }\end{array}$ & $\begin{array}{c}\text { Talla } \\
\text { mínima }\end{array}$ & Curtosis \\
\hline Machos Sexado & $142.84 \pm 59.11$ & 41.11 & 111.8 & 355.8 & 13.0 & $0.19(-)$ \\
Hembras Sexado & $93.05 \pm 39.55$ & 42.50 & 88.4 & 328.7 & 5.4 & 1.99 \\
\hline
\end{tabular}

\section{Engorde por sexo separado}

Hacia el final del ciclo de engorde no se observó en los machos una concentración de pesos para un rango específico de talla, mientras que para las hembras se anotó una concentración de pesos entre los 262.3 g y 342.3 g, que representó el 15.9\% de la población de hembras (figura 6).

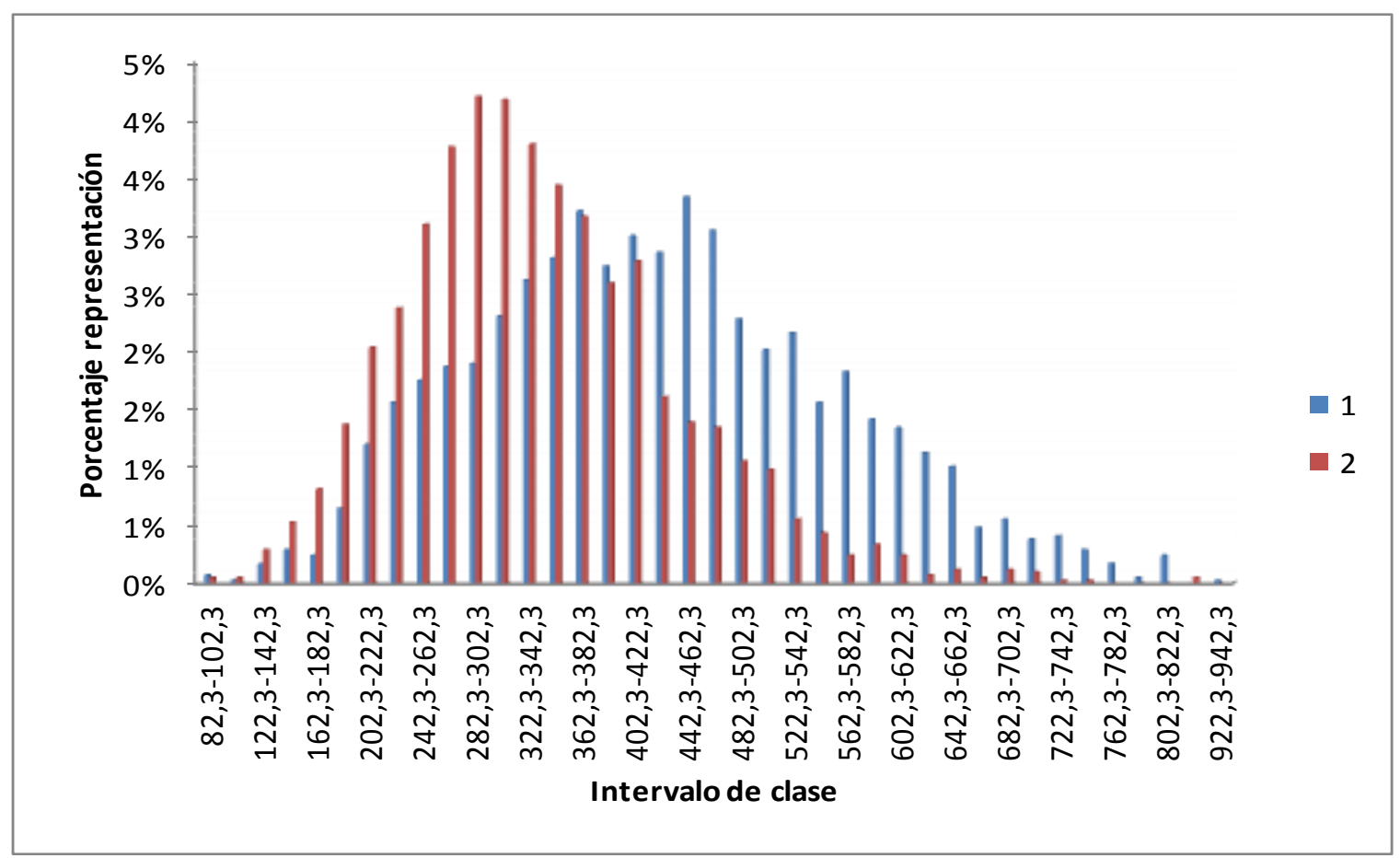

Figura 6.

Distribución de peso de machos (1) y hembras (2) al final del ciclo de engorde, peso promedio final para ambos sexos de $339.6 \mathrm{~g}$. 
El peso promedio de machos al final de la etapa de engorde registró un valor de $429.25 \pm 135.18 \mathrm{~g}$; su peso resultó superior al de las hembras que registraron $339.65 \pm 102.64$ g. Esta diferencia de peso representó una disminución del $20.9 \%$ del peso de las hembras con respecto al de los machos. El CV para los machos durante la fase de engorde por sexo separado fue de $31.49 \%$, lo cual evidencia una mejora en la distribución de pesos; el valor de curtosis negativo para los machos de -0.26 (Platicurtica) mostró que la curva de distribución de pesos fue más amplia (tabla 2).

Tabla 2

Resumen estadístico del peso de las hembras (sexado/engorde)

\begin{tabular}{lcccccc} 
Tipo de población & $\begin{array}{c}\text { Peso promedio en } \\
\text { gramos } \pm \mathrm{DE}\end{array}$ & $\mathrm{CV}$ & Moda & $\begin{array}{c}\text { Talla } \\
\text { máxima }\end{array}$ & $\begin{array}{c}\text { Talla } \\
\text { mínima }\end{array}$ & Curtosis \\
\hline Machos engorde & $429.25 \pm 135.18$ & 31.49 & 364.3 & 926.6 & 82.3 & $0.26(-)$ \\
Hembras engorde & $339.65 \pm 102.64$ & 30.22 & 365.0 & 833.8 & 83.8 & 1.05 \\
\hline
\end{tabular}

El CV para las hembras al final de la fase de engorde fue de $30,22 \%$, valor que indica una mejora en la dispersión de tallas con respecto a su valor inicial (42.50\% sexado) y con respecto a los machos (31.49\%), el valor positivo de curtosis de las hembras de 1.05 fue un indicador de una distribución elevada (Leptocurtica).

\section{Diferencial de peso y crecimiento machos-hembras}

La diferencia de peso de machos y hembras (429.3 vs $339.7 \mathrm{~g}$ ) representa una diferencia del $26.3 \%$ de superioridad del macho con respecto a la hembra, mientras que la tasa de crecimiento (2.73 g día ${ }^{-1}$ vs $\left.2.17 \mathrm{~g} \mathrm{día}^{-1}\right)$ muestra una diferencia de crecimiento 0.56 gramos diarios para un nivel de $20.51 \%$ de superioridad del crecimiento de los machos (tabla 3).

Tabla 3

Comparación del crecimiento en $g$ día ${ }^{-1}$ entre machos y hembras durante el engorde

\begin{tabular}{lcccc} 
& $\begin{array}{c}\text { Tasa } \\
\text { crecimiento } \\
\left(\mathrm{g} \mathrm{día}^{-1}\right)\end{array}$ & $\begin{array}{c}\text { Días } \\
\text { ciclo }\end{array}$ & $\begin{array}{c}\text { Diferencia } \\
\text { crecimiento } \\
\left(\mathrm{g} \mathrm{día}^{-1}\right)\end{array}$ & $\begin{array}{c}\% \\
\text { diferencia }\end{array}$ \\
\hline Machos & 2.73 & 150 & 0.56 & 20.51 \\
\hline Hembras & 2.17 & & & \\
\hline
\end{tabular}


Uniciencia. Vol. 29, No. 1, pp. 1-15. Enero, 2015.

ISSN Electrónico: 2215-3470

URL: www.revistas.una.ac.cr/uniciencia

Email: revistauniciencia@una.cr

DOI: http://dx.doi.org/10.15359/ru.29-1.1

Diferencias estadísticas a un nivel del $5 \%$ fueron determinadas para los parámetros peso de cosecha y tasa de crecimiento entre hembras y machos: ambos parámetros superiores en los machos (tabla 4).

Tabla 4

Valores de peso de cosecha y crecimiento final para machos-hembras engordados en jaulas y por sexo separado

\begin{tabular}{ccc}
\hline Rango de Peso $(\mathrm{g})$ & \multicolumn{2}{c}{$26.2-387.0$} \\
\hline Sexo de tilapias & $\begin{array}{c}\text { peso promedio en } \\
\text { gramos } \pm \mathrm{DE}\end{array}$ & $\begin{array}{c}\text { Tasa de crecimiento } \\
\text { promedio g día }\end{array}{ }^{-1} \pm \mathrm{DE}$ \\
\hline Machos & $429.3 \pm 135.2^{\mathrm{a}}$ & $2.74 \pm 0.86^{\mathrm{a}}$ \\
Hembras & $339.7 \pm 102.6^{\mathrm{b}}$ & $2.18 \pm 0.68^{\mathrm{b}}$ \\
\hline
\end{tabular}

Promedio ( \pm s.d.) con diferente letra superscrita, dentro de la columna de datos indica diferencia estadística significativa a un nivel de significancia del $5 \%$.

La estimación de la diferencia de pesos entre machos y hembras de tilapia, para un intervalo de confianza de $95 \%$ fue de $89.62 \mathrm{~g}$ (tabla 5).

Tabla 5

Estimación de la diferencia de pesos entre machos y hembras a peso de cosecha

\begin{tabular}{cccc} 
& $\begin{array}{c}\text { Peso } \\
\text { promedio }(\mathrm{g})\end{array}$ & $\begin{array}{c}\text { Error } \\
\text { estándar }\end{array}$ & $\begin{array}{c}\text { Diferencia } \\
\text { pesos }(\mathrm{g})\end{array}$ \\
\hline Macho & 429.3 & 8.26 & $89.62 \pm 7.23$ \\
Hembra & 339.7 & 5.33 & \\
\hline
\end{tabular}

\section{Discusión}

Los machos de tilapia se caracterizan por presentar un crecimiento superior que las hembras (Toguyeni et al., 1996). Las diferencias de tamaño y crecimiento entre machos y hembras de tilapia son afectadas por el proceso de maduración sexual (Bwanika et al., 2007). Para $O$. mossambicus se determinó que el mayor crecimiento experimentado por los machos se correlaciona con el inicio de la espermiogénesis, mientras que en las hembras el menor crecimiento se vio influenciado por el proceso de ovogénesis (Bhatta et al., 2012).

Mediciones de crecimiento entre machos y hembras de la tilapia O. niloticus tanto en líneas puras (LP) como en líneas mejoradas genéticamente (LM) han reflejado diferencias en crecimiento. Para machos y hembras de O. niloticus (LP) con pesos de siembra de $24.7 \mathrm{~g}-28.6 \mathrm{~g}$ y de cosecha de $230.0 \mathrm{~g} 198.6 \mathrm{~g}$ se determinaron diferencias significativas para el peso de cosecha y para la tasa de crecimiento absoluto $\left(0.57 \mathrm{~g} \mathrm{día}^{-1}\right.$ 
vs $0.47 \mathrm{~g} \mathrm{día}^{-1}$ ), los machos mostraron una mayor superioridad (Sule, 2004). Más recientemente en $O$. niloticus (LM) se evaluó el peso de cosecha y tasa de crecimiento de individuos cultivados por sexo separado y se lograron obtener diferencias significativas para peso de cosecha $(628.4 \mathrm{~g}-408.0 \mathrm{~g})$ y para la tasa de crecimiento absoluto $\left(3.19 \mathrm{~g}\right.$ día ${ }^{-1}$ vs $1.93 \mathrm{~g} \mathrm{día}^{-1}$ ) para machos y hembras, respectivamente: los machos lograron mejor desempeño productivo (Ridha, 2011).

En esta investigación se determinaron, para machos y hembras, diferencias significativas para el peso de cosecha (429.2 g - $339.65 \mathrm{~g})$, y para la tasa de crecimiento absoluto (2.74 $\mathrm{g} \mathrm{día}^{-1}$ y $\left.2.18 \mathrm{~g} \mathrm{día}^{-1}\right)$, y los machos resultaron superiores en cuanto a su crecimiento, lo que concuerda con los resultados obtenidos por Sule (2004) y Ridha (2011).

Un diferencial de crecimiento de 89.62 g en los machos representaría una extensión en el ciclo de cultivo de las hembras de aproximadamente 41 días, tiempo requerido para igualar la talla de cosecha de los machos; no obstante, esta prolongación de tiempo podría verse compensada con la utilidad alcanzada en la no compra de alevines por parte de productores que no tienen disponibles alevines sexo-reversados -ya sea por la lejanía de sus centros de cultivo o debido a que el costo relacionado con la compra y traslado de alevines dificulta la implementación del engorde de tilapias-. El engorde de las hembras podría compensar los costos de adquisición de alevines de proveedores externos y el mismo productor sería quien realice la reproducción de tilapias, sin la necesidad de aplicar técnicas de reversión sexual mediante hormonas, para posteriormente, durante el engorde, separar manualmente los machos de las hembras y cultivarlas separadamente.

\section{Referencias}

Alicorp, S.A. (2004). Manual de crianza de tilapia. Lima, Perú. Recuperado de http://www.alicorp.com.pe.pdf

Bhatta, S., Iwai, T., Miura, T., Higuchi, M., Maugar, G., \& Miura, C. (2012). Differences between male and female growth and sexual maturation in tilapia (Oreochromis mossambicus) [Las diferencias entre macho y hembra en el crecimiento y la maduración sexual en tilapia (Oreochromis mossambicus)]. Journal of Science, Engineering and Technology, Kathmandu University, 8(2), 57-65.

Bhujel, R. C. (2000). A review of strategies for the management of Nile tilapia (Oreochromis niloticus) broodfish in seed production systems, especially hapa-based systems [Una revisión de las estrategias para el manejo de la tilapia del Nilo (Oreochromis niloticus) reproductores en sistemas de producción de semillas, sistemas especialmente hapa-basado]. Aquaculture 181, 37-59. http://dx.doi.org/10.1016/S0044-8486(99)00217-3

Buysee, W., Stern, R., Coe, R., \& Matere, C. (2008). GenStat Discovery Edition 3 [Genstat Descubrimiento Edición 3], 2000. Edición para uso cotidiano. (Trad. José Rodolfo García Nava, Victorino Morales Ramos, Martha Elva Ramírez Guzmán) Nairobi: ICRAF.

Bwanika, G. N., Murie, D. J., \& Chapman, L. J. (2007). Comparative age and growth of Nile tilapia (Oreochromis niloticus L.) in lakes Nabugabo and Wamala, Uganda [comparativo entre 
Uniciencia. Vol. 29, No. 1, pp. 1-15. Enero, 2015.

URL: www.revistas.una.ac.cr/uniciencia

Email: revistauniciencia@una.cr
ISSN Electrónico: 2215-3470

DOI: http://dx.doi.org/10.15359/ru.29-1.1

edad y el crecimiento de la tilapia del Nilo (Oreochromis niloticus L.) en los lagos Nabugabo y Wamala, Uganda]. Hydrobiologia 589(1), 287-301. http://dx.doi.org/10.1007/s10750-007-0746-y

Chakraborty, S. B., \& Banerjee, S. (2010). Comparative growth performance of mixed-sex and monosex Nile Tilapia population in freshwater cage culture system under Indian perspective [El crecimiento comparativo de sexos-mistos y mono-sexos en la población de tilapia del Nilo en agua dulce sistema de cultivo en jaulas en perspectiva de la India]. International Journal of Biology, 2(1), 44-50. http://dx.doi.org/10.5539/ijb.v2n1p44

Dunham, R. A., Majumdar, K., Hallerman, E., Bartley, D., Mair, D., Hulata, D., Pongthana, L.N., Bakos, J., Penman, D., Gupta, M., Rothlisberg, P., \& Hoerstgen, G.S. (2000). Review of the Status of Aquaculture Genetics [Revisión de la Condición de la Acuicultura Genética] . In: Aquaculture in the Third Millennium Technical Proceedings of Conference on Aquaculture Bangkok, Thailand, FAO, Roma.

Gjedrem, T. (2005). Selection and Breeding Programs in Aquaculture. [Programas de selección y mejora genética en acuicultura]. Netherlands: Springer. http://dx.doi.org/10.1007/1-4020$\underline{3342-7}$

Hopkins, K. D. (1992). Reporting Fish Growth: A review of the Basics Journal of the World[Informes crecimiento de los peces: Una revisión de la literatura básica Mundial. Aquaculture Society, 23(3), 173-179. http://dx.doi.org/10.1111/j.1749-7345.1992.tb00766.x

Mair, G. C. (1995). Genetic manipulation for improved tilapia (GMIT) Technology Adaptation and Development I. [La manipulación genética de la tilapia mejorada (GMIT) Tecnología de Adaptación y Desarrollo I]. ODA Fish Genetic Program (R5068A), Thailand.

Nunes, A. J. (2010). Tilapia Cage Farm Management in Brazil. [Administración de granjas de tilapia en Brasil]. Global aquaculture advocate. Descargado de http://www.thefishsite.com/articles/1014/tilapia-cage-farm-management-in-brazil

Ridha, M. T. (2011). Evaluation of monosex culture of GIFT and non-improved strain of Nile Tilapia Oreochromis niloticus in recirculating tank[Evaluación de la cultura monosex de GIFT y de cepa no mejorada de tilapia del Nilo Oreochromis niloticus en el tanque de recirculación]. International Aquatic Research 3, 189-195.

Ridha, M. T., \& Cruz, E.M. (1998). Observations on the seed production of the tilapia Oreochromis spilurus (Gunter) under different spawning condition and with different sex ratios. [Observaciones sobre la producción de la tilapia Oreochromis spilurus (Gunter) bajo diferentes condiciones de desove y con distintas relaciones sexuales.]. Asian Fisheries Science, 10, 201-210.

Saavedra, M. M. (2006). Manejo del cultivo de tilapia. Descargado de http://csptilapianayarit.org/informacion/Generalidades_del_cultivo_de_Tilapia.pdf

Salvador, F. M., \& Gallardo, P. (2003). Análisis exploratorio de datos. Recuperado de www.ciberconta.unizar.es/Leccion/aed/ead.pdf 
Uniciencia. Vol. 29, No. 1, pp. 1-15. Enero, 2015.

ISSN Electrónico: 2215-3470

URL: www.revistas.una.ac.cr/uniciencia

Email: revistauniciencia@una.cr

DOI: http://dx.doi.org/10.15359/ru.29-1.1

Schmittou, H. (1991). Guidelines for Raising Principally Omnivorous Carps, Catfishes and Tilapias in Cages Suspended in Freshwater Ponds, Lakes and Reservoirs. [Directrices para Elevar Principalmente Omnívoro carpas, bagres y tilapias, en jaulas suspendidas en agua dulce Estanques, lagos y embalses]. Proceedings of the People's Republic of China Aquaculture and Feed Workshop [Taller de la República de China sobre Acuicultura y alimentos], D. Akiyama, (Ed.). Singapore: American Soybean Association.

Sule, O. D. (2004). Growth performance of mono-sex and mixed sex population of Oreochromis niloticus fed similar diet. [Los resultados de crecimiento de mono-sexo y población de ambos sexos de Oreochromis niloticus alimentada con dieta similar.]. Animal Research International, 1(2), 106-109.

Toguyeni, A., Baroiller, J.F., Fostier, A., Le Bail, P.Y., Khün, E.R., Mol, K.A., \& Fauconneau, B. (1996). Consequences of food restriction on shortterm growth variation and plasma circulating hormones in Oreochromis niloticus in relation to sex. [Consecuencias de la restricción de alimentos en la variación del crecimiento a corto plazo y el plasma que circula hormonas en Oreochromis niloticus en relación al sexo.]. General and Comparative Endocrinology, 103, 167-175 http://dx.doi.org/10.1006/gcen.1996.0107

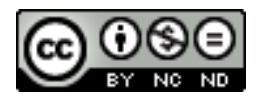

Comparación del crecimiento de machos y hembras de la tilapia Oreochromis Niloticus cultivadas en jaulas (Carlos Alvarado Ruiz) por Revista Uniciencia se encuentra bajo una Licencia CreativeCommons Atribución-NoComercialSinDerivadas 3.0 Unported. 